\title{
Error estimation for adaptive computations of shell structures
}

\author{
Pedro Díez \& Antonio Huerta \\ Universitat Politècnica de Catalunya \\ E.T.S. Ingenieros de Caminos Barcelona \\ Campus Nord, E-08034 Barcelona (Spain) \\ pedro.diez@upc.es
}

ABSTRACT. The finite element discretization of a shell structure introduces two kinds of errors: the error in the functional approximation and the error in the geometry approximation. The first is associated with the finite dimensional interpolation space and is present in any finite element computation. The latter is associated with the piecewise polynomial approximation of a curved surface and is much more relevant in shell problems than in any other standard $2 D$ or $3 D$ computation. In this work, a residual type error estimator introduced for standard finite element analysis is generalized to shell problems. This allows easily to account for the real original geometry of the problem in the error estimation procedure and precludes the necessity of comparing generalized stress components between non coplanar elements. That is, the main drawbacks of flux projection error estimators are avoided.

RÉSUMÉ. La discrétisation par éléments finis d'une coque introduit deux types d'erreurs différentes: l'erreur dans l'approximation fonctionnelle et l'erreur dans l'approximation de la géométrie. La première est associée à l'espace d'interpolation qui est de dimension finie et apparaît dans n'importe quel calcul par éléments finis. La deuxième est liée à remplacer la surface courbe de la coque par un domaine polyédrique. Ce phénomène est beaucoup plus important dans les coques que dans les problèmes standard 2-D ou 3-D. Cet article présente la généralisation aux éléments de coques d'un estimateur d'erreur de type résiduel qui avait été introduit pour des éléments finis standard. Cet estimateur permet de tenir compte dans le processus d'estimation de l'erreur de la géométrie du problème continu originel. Il permet aussi d'éviter la comparaison des composantes des contraintes généralisées entre des éléments non coplanaires, dont les estimateurs de projection de flux ne savent pas s'en passer.

KEYWORDS: Error estimation, adaptivity, thin shell elements, locking free formulation

MOTS-CLÉS : Estimation de l'erreur, adaptivité, éléments de coquemince, formulation sans verrouillage. 


\section{Introduction}

Structural elements, that is beams, shells or plates, are used to model the behavior of 3D structures with one dimension much larger (beams) or much smaller (plates and shells) than the other two. For instance, in shells, the thickness is negligible in front of the dimensions of the midsurface. If the Reissner-Midlin theory is adopted, the displacements in every point of the shell are described through the displacements of the points lying on the midsurface and the rotation of the cross section. The domain of the mathematical problem to solve is therefore a surface instead of a volume. In fact, the equilibrium equation is adanted for this kinematic descrintion and the resulting problem is a PDE for which the unknown is a displacement-rotation field (6 d.o.f.) taking values on a 2D manifold (a surface). Paradoxically, the kinematic description is derived assuming that the thickness is small enough but this formulation locks when the thickness tends to zero. Consequently, the formulations of thin shell finite elements have to be manipulated in order to avoid shear locking [AYA 98]. These manipulations are often introduced in the discrete form of the problem[DON 87] and, therefore, they lead to a discrete equation which is not derived from a continuous weak form. In fact, for this kind of problem, the formulation is intrinsically discrete, that is, there is no

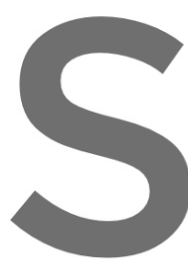
continuous form of th

\section{written in th}

of a continu

possible to defin

approximate se

rationale, the use of res
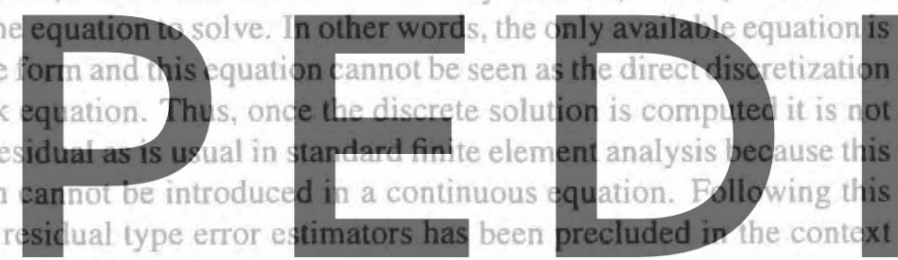

of shell formulations [LEE 99].

Register for free at httpspawww.seipediandonf to dow rildad the version without the watermark projection error estimates, see [BAU 97, CIR 98, RIC 97]. Flux projection error estimates have become very popular due to their simplicity and robustness. Nevertheless, they iack a sound theoreticai background and are difficuit to justify in the absence of superconvergent phenomena. In the shell context two additional drawbacks must be pointed out: 1) the flux smoothing averages stress components over different elements that may have different physical meaning if the tangent planes are different, and 2) the error estimation process uses only the approximate solution and hence, the discretized forces and the computational mesh: the data describing the real geometry and load is therefore not accounted for. This implies, for example, that the flux projection error estimate cannot account for the error associated with the geometrical discretization, that is, the effect of replacing the real geometry of the structure by a finite element mesh.

Thus, in this work a residual type estimator [DIE 98] is generalized for shell elements in order to obtain reliable estimates and to drive adaptive procedures. As previously stated, the use of residual type error estimators must be carefully handled because there is no available residual equation for the error. In fact, this is due to the interpolation tricks used in the shell formulation to avoid shear locking because these tricks are formulated at the discrete level. Then, the computation of the residual in the 
local error equations that has to be solved in the error estimation strategy requires a special treatment. The main idea is to introduce a reference solution associated with an $h$-refined mesh and to define a residual in this "almost continuous" equation. Attention must be paid, then, to the transfer of data from the rough computational mesh to the refined reference mesh. Moreover, the geometry of the mesh is also approximated by the finite element mesh. In standard $2 \mathrm{D}$ or $3 \mathrm{D}$ problems the geometric approximation affects only the boundary of the domain but in structural shell elements this affects the whole domain. Thus, it can be stated that the finite element discretization induces two sources of error:

- The error in the functional approximation is related to the approximation of the infinite dimensional functional space to whom the solution belongs by a finite dimensional interpolation space induced by the mesh. This source of error is present in any finite element computation and the usual error estimators are designed to account for it.

- The error in the geometric approximation is only relevant in the shell context and the generalization of the error estimator must be carefully designed to account for it. In fact, flux projection error estimates use only the approximate solution to compute

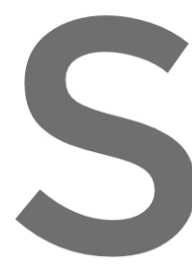
the error esimate and projection geometry.

\section{The remainder of $\mathrm{t}$}
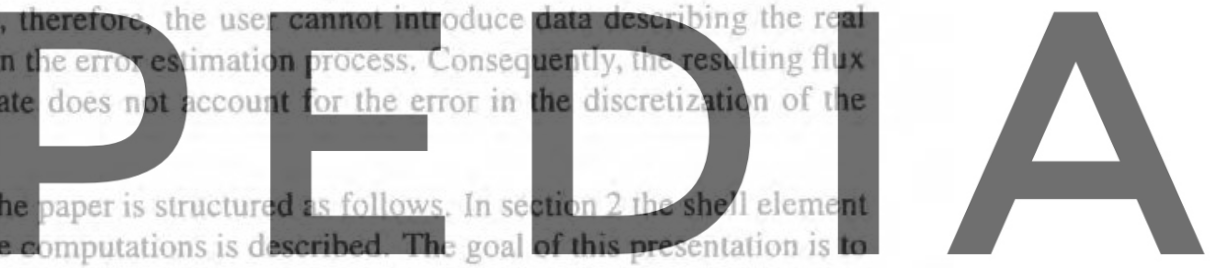

highlight the interpolation tricks, used to preclude the shear locking, that affect the

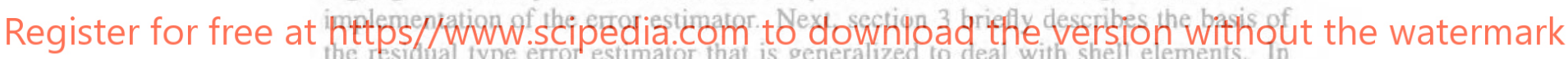
section 4 a strategy to transfer data from one mesh to another in the context of the sheil formuiation is intoduced. This strategy is required to compute the residual in the local error equations but can also be used for other purposes. Finally, in section 5 numerical examples demonstrating the effectiveness of the error estimation procedure and its capability to drive adaptive procedures are shown.

\section{Shell element formulation}

This section introduces the main characteristics of the shell formulation that has been selected in this work. The main objective of this presentation is to introduce notation and to emphasize the particularities of the interpolation that are introduced to avoid shear locking. These particularities affect the implementation of the error estimator described in section 3.

The Reissner-Midlin theory for plates and shells decouples the rotation of the plate (or shell) cross section from the slope of the midsurface. This allows to take into account shear deformation and only requires $\mathcal{C}^{0}$ kinematic continuity. The unknowns 
of the Reissner-Midlin formulation are the displacement of the midsurface points, $u_{x}$, $u_{y}$ and $u_{z}$, and the rotation of the cross section, $\theta_{x}, \theta_{y}$ and $\theta_{z}$. Thus, the displacement of every point of the shell is recovered from these six values.

Contrary to the Kirchhoff formulation that neglects the effect of transverse shear and requires the use of $\mathcal{C}^{1}$ interpolates, the Reissner-Midlin theory behaves well for moderately large or large thicknesses but exhibits a severe degradation of accuracy when the span-to-thickness ratio becomes very large. This numerical pitfall is known as transverse shear element locking.

Many authors have proposed solutions to overcome the appearance of shear locking in beams, plates and shells, see reference [AYA 98] for a short review. In this work, the shell formulation introduced by Donea and Lamain [DON 87] is used to test the capability of the proposed error estimation approach to be generalized to structural shell problems. This formulation uses a particular interpolation of the rotation terms that must be carefully treated in the implementation of the error estimator. A. discussion on the selection of the optimal formulation for the shell problem is out of the scope of this paper.
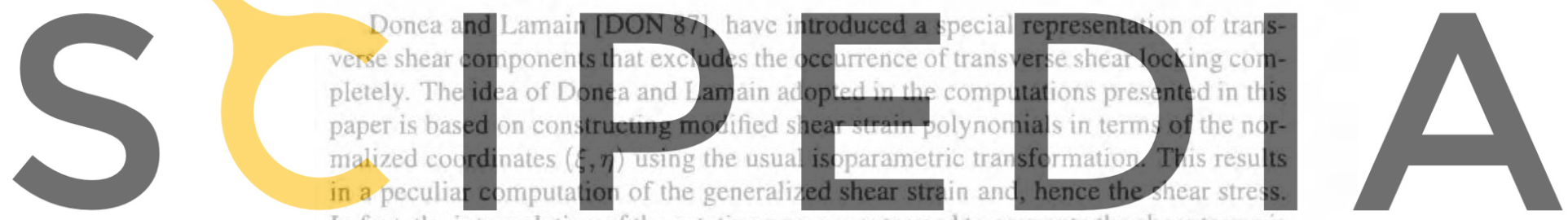

In fact, the interpolation of the rotation components used to compute the shear terms is different from the isoparametric interpolation that is still used to compute the bending

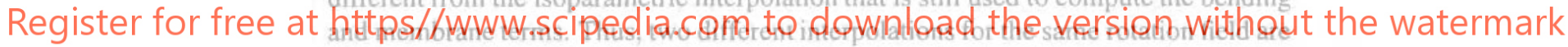
used simultaneously in the same element. That results in a non-unique description of the rotations inside the elements.

Let us illustrate the modifications imposed on a shear component in the particular case of a plane element in the $O x y$ plane. The shear strain $\gamma_{x}$ is expressed by

$$
\gamma_{x}:=\frac{\partial u_{z}}{\partial x}-\theta_{y} .
$$

The usual isoparametric interpolations for the displacements and rotations read

$$
u_{\alpha}(\xi, \eta)=\sum_{i=1}^{n_{\mathrm{cn}}} N_{i}(\xi, \eta) u_{\alpha}^{i} \text { and } \theta_{\alpha}(\xi, \eta)=\sum_{i=1}^{n_{\mathrm{en}}} N_{i}(\xi, \eta) \theta_{\alpha}^{i},
$$

where $n_{\mathrm{en}}$ is the number of element nodes, $N_{i}(\xi, \eta)$ are the normalized shape functions and $\alpha=x, y, z$. Then, replacing Eq. [2] in Eq. [1]

$$
\gamma_{x}:=\sum_{i=1}^{n_{\mathrm{ea}}}\left(\frac{\partial N_{i}}{\partial x} u_{z}^{i}-N_{i} \theta_{y}^{i}\right) .
$$


and, for instance, $P_{\xi}$ is modified such that

$$
\begin{aligned}
P_{\xi}= & \frac{1}{4}(1-\eta)\left(u_{z}^{2}-u_{z}^{1}\right)+\frac{1}{4}(1+\eta)\left(u_{z}^{3}-u_{z}^{4}\right) \\
& -\frac{1}{8}(1-\eta)\left(x_{2}-x_{1}\right)\left(\theta_{y}^{1}+\theta_{y}^{2}\right)-\frac{1}{8}(1+\eta)\left(x_{3}-x_{4}\right)\left(\theta_{y}^{3}+\theta_{y}^{4}\right) \\
& -\frac{1}{8}(1-\eta)\left(y_{2}-y_{1}\right)\left(\theta_{x}^{1}+\theta_{x}^{2}\right)-\frac{1}{8}(1+\eta)\left(y_{3}-y_{4}\right)\left(\theta_{x}^{3}+\theta_{x}^{4}\right) .
\end{aligned}
$$

Note that the second and third line of Eq. $\{11\}$ correspond to the terms $\frac{\partial x}{\partial \xi} \theta_{y}$ and $\frac{\partial y}{\partial \xi} \theta_{x}$, which appear in the definition of $P_{\xi}$, see Eq. [9]. Note also that the unmodified polynomials described in Eq. [9] are of order three and the modified $P_{\xi}$ of Eq. [11] is of order one. This implies that the interpolation of the modified shear stresses (multiplied by $|\mathbf{J}|$ ) are, in this case, assumed to be also of order one, see Eq. [8].

As it is pointed out in section 3, the error estimation procedure that is going to be generalized to shell elements in this work [DIE 98] requires to obtain values of the approximate solution $u_{h}$ in a number of nodes inside each element of the computa-

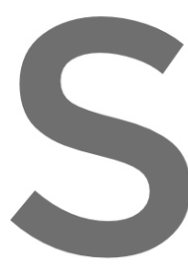
tional mesh. using the us tion forbids the generali transfer the finit technique is in roduced in section 4.
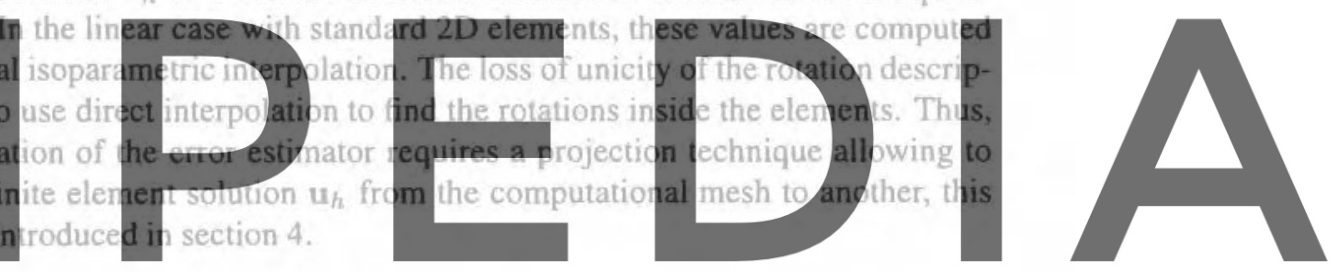

Register for free at httpsfifwwwseipedia.com to download the version without the watermark

The residual type error estimator that is generalized in this work has been first introduced for 2D standard linear finite element analysis [DIE 98]. The same idea has also been used for nonlinear analysis in [DIE 00, HUE 97, HUE 00]. The efficiency of the estimator has been studied in [DIE 97].

This estimator is based on the definition of a reference solution associated with a uniformly refined reference mesh. Each element of the computational mesh (of characteristic size $h$ ) is refined using a local elementary submesh. The reference mesh is the assembly of all the local elementary submeshes, see figure 1 for an illustration. The characteristic size of the reference mesh is denoted by $\tilde{h}(\bar{h}<<h)$. In the following the objects marked with the subscripts $h$ and $\tilde{h}$ are associated with the computational mesh and the reference mesh respectively. Thus, a reference solution $\mathbf{u}_{\bar{h}}$ and a reference error $\mathbf{e}_{\bar{h}}:=\mathbf{u}_{\bar{h}}-\mathbf{u}_{h}$ are introduced. The reference error is the solution of the following problem, associated with the fine reference mesh:

$$
\mathbf{K}_{\tilde{h}} \mathbf{e}_{\tilde{h}}=\mathbf{r}_{\tilde{h}}\left(\mathbf{u}_{h}\right) \text {, }
$$

where $\mathbf{K}_{\bar{h}}$ is the stiffness matrix in the reference mesh and $\mathbf{r}_{\bar{h}}\left(\mathbf{u}_{h}\right)$ is the residual associated with $\mathbf{u}_{h}$. Note that $\mathbf{e}_{\bar{h}}$ is not the error of $\mathbf{u}_{\bar{h}}$ but the error of $\mathbf{u}_{h}$ with respect 
Error estimation for shell structures

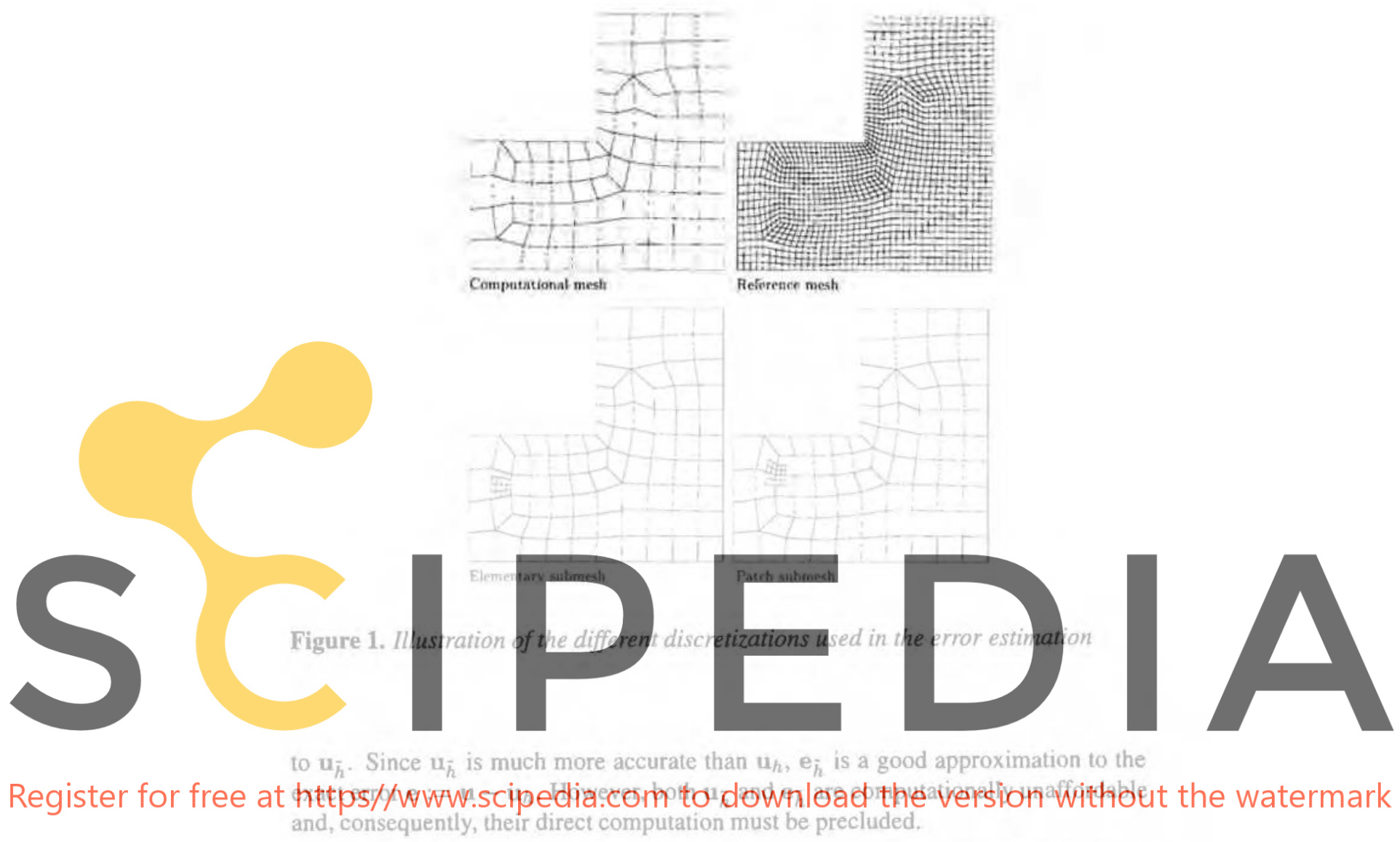

The idea of the estimator is to replace the direct computation of $\mathrm{e}_{\bar{n}}$ (or $u_{\bar{n}}$ ), which requires the solution of the linear system of Eq. [12], that is a problem associated with the reference mesh, by a set of local problems, associated with the elementary submeshes. Instead of deriving boundary conditions for the local problems using a standard flux splitting procedure, trivial homogeneous Dirichlet boundary conditions are imposed. That leads to a first estimate describing the part of the error associated with the interior of the elements. This part of the error ignores the contribution of the flux jumps across the element edges. A new set of subdomains denoted by patches is defined such that they cover the original domain overlapping the elements, see figure 1 . A new set of problems, one over each patch, is solved and a new contribution to the error is obtained. Each local component of the error is obtained by solving a local equation homologous to Eq. [12]. It is worth noting that computing the patch estimates an additional linear restriction must be added to the local problem in order to ensure orthogonality with respect to the interior estimate. This allows to properly add the interior and patch estimates.

Thus, the error estimate is computed in two phases. The first phase accounts for the error related to the interior (smooth) residual and the second phase accounts for the 
error associated with the flux jumps (singular residual), see [HUG 98] for a clarifying discussion on the two sources of error.

Two problems arise to generalize this estimator to the context of shell elements: the computation of the residual and the possibility of taking into account the real geometry of the shell.

\subsection{Difficulties in the computation of the residual}

Every local problem to be solved requires to compute the residual as the independent right-hand-side term of the local (element by element or patch by patch) counterpart of the error equation Eq. [12]. The residual $\mathbf{r}_{\bar{h}}\left(\mathbf{u}_{h}\right)$ of the approximate solution $\mathbf{u}_{h}$ associated with the (or expressed in) the fine reference mesh reads

$$
\mathbf{r}_{\bar{h}}\left(\mathbf{u}_{h}\right):=\mathbf{f}_{\bar{h}}^{\text {ext }}-\mathbf{f}_{\bar{h}}^{\text {int }}\left(\mathbf{u}_{h}\right) \text {, }
$$

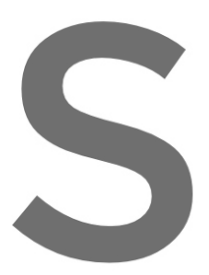

where $\mathrm{f}_{\bar{h}}^{\text {ext }}$ is the discretization of the external forces in the reference mesh and $\mathrm{f}_{\bar{h}}^{\text {int }}\left(\mathrm{u}_{h}\right)$
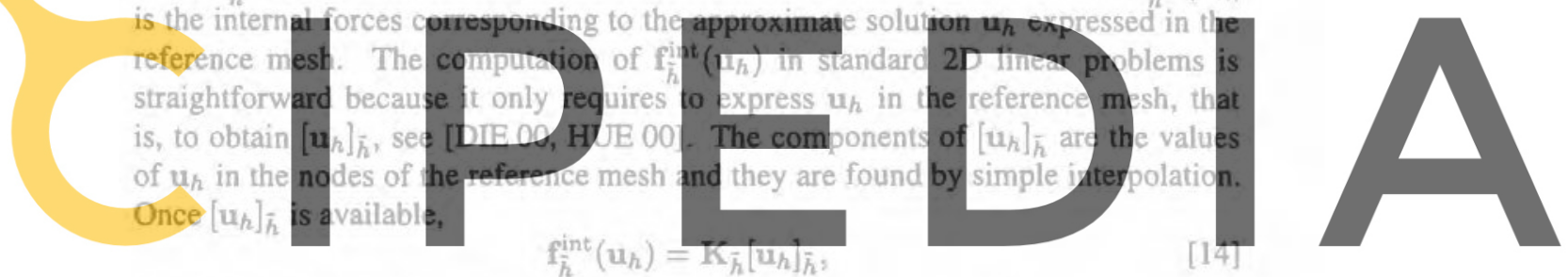

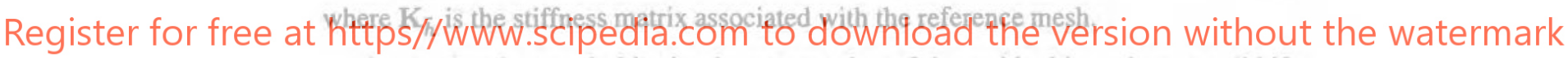

As previously stated, this simple computation of the residual is no longer valid if the shell elements described in section 2 are used. This is because the components of $\left[\mathbf{u}_{h}\right]_{\tilde{h}}$ corresponding to the rotation terms cannot be interpolated using a unique description. The problem, now, is how to obtain $\mathbf{f}_{\tilde{h}}^{\text {int }}\left(\mathbf{u}_{h}\right)$ precluding the computation of $\left[\mathbf{u}_{h}\right]_{\bar{h}}$ (the simple isoparametric interpolation). Once $\mathbf{f}_{\tilde{h}}^{\text {int }}\left(\mathbf{u}_{h}\right)$ is found, a displacement-rotation field over the reference mesh, $\left[\mathbf{u}_{h}\right]_{\hat{h}}$, may be recovered solving Eq. [14]. This is described is section 4 and, in fact, it provides a general metodology to transfer the solution from one mesh to another in the shell elements context.

\subsection{Accounting for the real geometry}

The problem of taking into account the real geometry in the error estimation process may be easily handled following the approach introduced in this section. It suffices to build up a proper reference mesh using the data describing the real surface of the shell.

The usual implementation of the presented error estimator defines the reference submesh as the assembly of all the elementary submeshes. Each of these elementary 

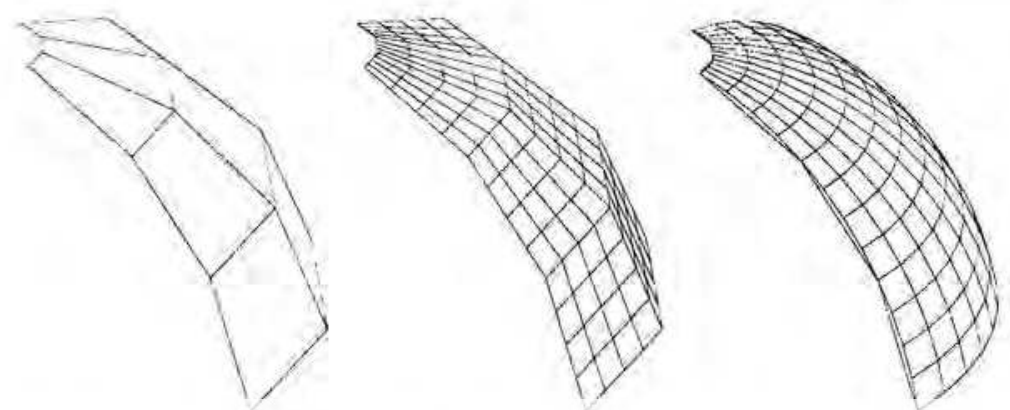

Figure 2. Computational mesh (left), reference submesh built up without real geometry data (center) and reference submesh built up with real geometry data (right).

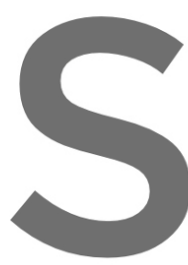

ubmeshes is a discretization of the corresponding element. However, using this approach in the shell case gives an unrealistic reference mesh, see figure 2 . Indeed, the mesh in the center in figure 2 is built up by simply refining each element of the left computational mesh. The only dax about the geometry of the shell that the been used to eonstruct this refined rnesh is the location of the nodes of the computational mesh. These nodes The rest of the etry of the eal geonietry, as shown in the right mesh in figure 2 . This nesh is buit up such that all its nodes are located on the real geometry of the shell, that is, using an Register for free at bttps/.WWWW.SGipedia.com to download the version without the watermark

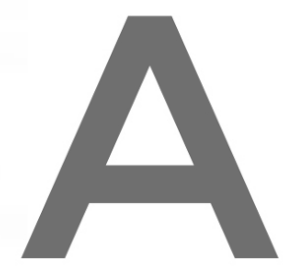

This process is illustrated in figure 3 ; a shell with curved geometry (figure 3 (a)) is computed with a computational mesh (figure 3 (b)) and the error affecting the approximate solution must be estimated. The error is estimated using a reference mesh fitting the real geometry (figure 3 (c)). This reference mesh is built up as the assembly of a number of elementary reference submeshes. The data describing the real geometry of the shell must be accounted for when constructing the elementary submeshes (figure 3 (d)).

\section{Transfer procedure}

As already mentioned in section 2 , the formulation used in this work to avoid shear locking in the thin shell elements, makes it difficult to transfer the information from one mesh to another. The problem is stated as follows: given a solution $\mathbf{u}_{h}$ in a mesh of characteristic size $h$ find the transferred solution $\left[\mathbf{u}_{h}\right]_{\tilde{h}}$ in the refined mesh of characteristic size $\vec{h}$. The problem is that the interpolation of the rotation terms is not unique: the rotations are interpolated using the standard isoparametric description to compute bending and axial terms and using a modified set of polynomials to compute 


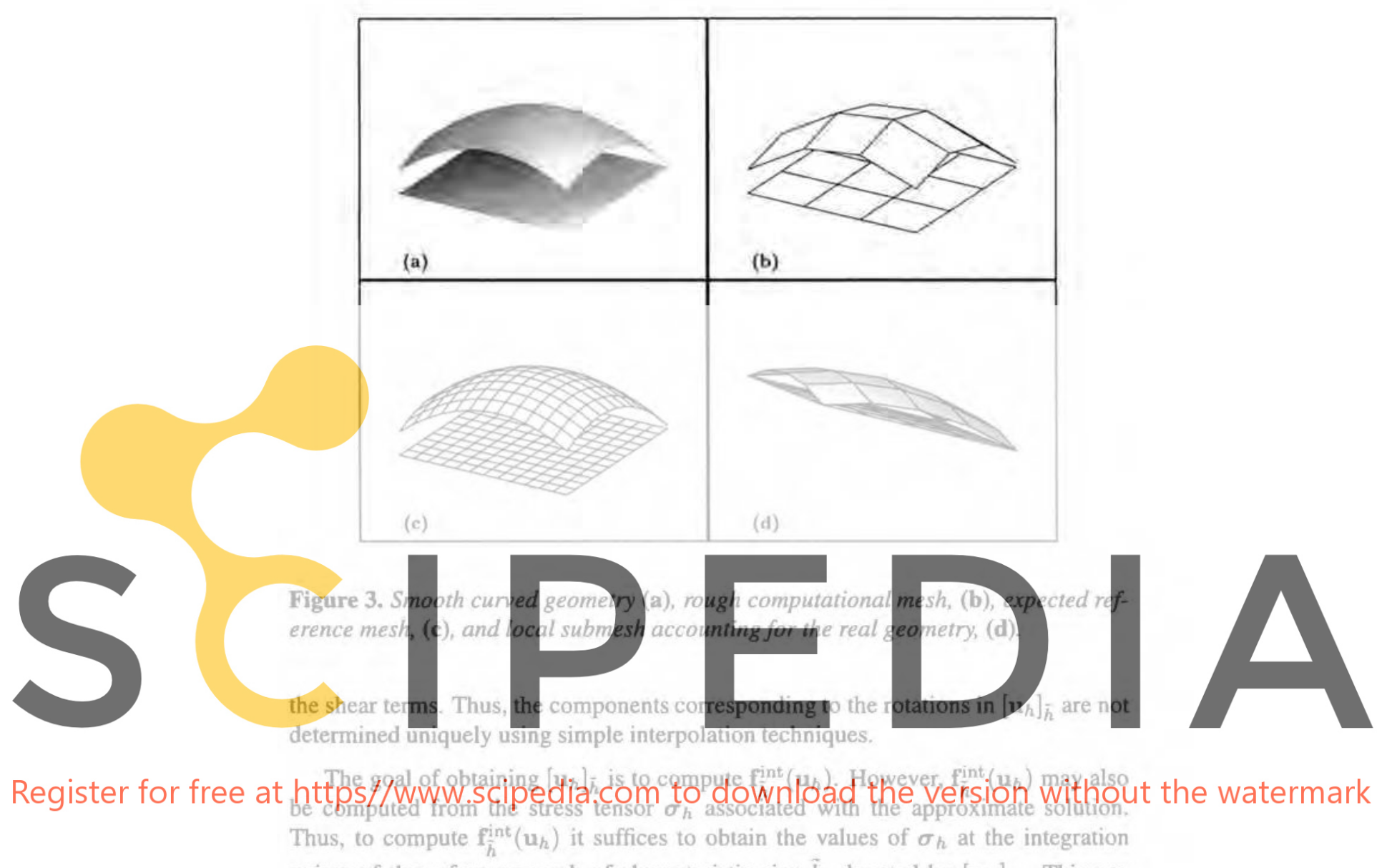

points of the reference mesh of characteristic size $\hat{h}$, denoted by $\left[\sigma_{h}\right]_{\tilde{h}}$. This can be done easily using simple pure interpolation techniques inside each element of the coarse computational mesh because, as stated is section 2, every stress component belongs to well known polynomial interpolation space (these spaces are different for each component). Once it is stated that every component of the generalized stresses has a unique representation in a given interpolation family, it is necessary to identify it.

In fact, as shown in section 2, the interpolation of the shear strain (or stress) terms (not the rotations) is prescribed using some modified polynomials, Eqs. (9] and [11]. Each component of the computed stress tensor $\sigma_{h}$ may be expressed inside an element by

$$
\left[\boldsymbol{\sigma}_{h}\right]^{j}=\frac{1}{\left|J_{h}(\xi, \eta)\right|} Q^{j}(\xi, \eta),
$$

where $\left[\sigma_{h}\right]^{j}$ is the $j$-th component of the generalized stress tensor and the polynomial $Q^{j}(\xi, \eta)$ belongs to a given interpolation space. This interpolation space of the stress components contains the proper derivatives of the shape functions if isoparametric 
interpolation is used, see Eq. [7], or is determined by the modifications introduced to preclude shear locking.

Then, the polynomial $Q^{j}(\xi, \eta)$ must be identified in the proper space using the known values of $\sigma_{h}$ (at the integration points of the coarse computational mesh). This can be done if the number of known values of the stress components inside each element is equal (or larger) to the number of degrees of freedom of the interpolation space to which $Q^{j}(\xi, \eta)$ belongs. For instance, in the case of the four-noded quadrilateral element, the bending and axial stress terms (multiplied by $|\mathbf{J}|$ ) which belong to a bilinear interpolation space are interpolated with the usual bilinear interpolation (four degrees of freedom). On the other hand, the shear terms (multiplied by $|\mathrm{J}|$ ) which are constant over the element are interpolated as a constant (one degree of freedom).

Thus, once $Q^{j}(\xi, \eta)$ is identified for every $j$, the stress terms are computed in all the integration points of the fine reference mesh of characteristic size $h$. This transferred stresses are denoted by $\left[\sigma_{h}\right]_{\bar{h}}$.

This process can be done elementwise because the reference mesh is a refinement of the computational mesh.
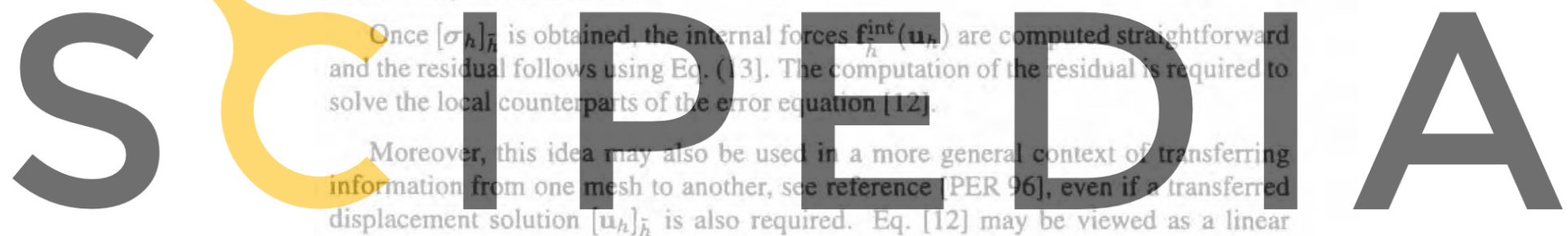

system of equations with unknown $\left[u_{h}\right]_{h}$.

Register for free at https//www.scipedia.com to download the version without the watermark $\mathrm{K}_{\bar{h}}\left[\mathrm{u}_{h}\right]_{\bar{h}}=\mathrm{f}_{\bar{h}}^{\mathrm{int}}\left(\mathrm{u}_{h}\right)$,

that can be solved once $\mathbf{f}_{\bar{h}}^{\text {int }}\left(\mathbf{u}_{h}\right)$ is computed. Note that the obtained $\left[\mathbf{u}_{h}\right]_{\bar{h}}$ is equivalent to $u_{h}$ in the energetic sense, that is, the energy norm of $\mathbf{u}_{h}$ is equal to the energy norm of $\left[\mathbf{u}_{h}\right]_{\bar{h}}$;

$$
\left\|\mathbf{u}_{h}\right\|^{2}=\mathbf{u}_{h}{ }^{T} \mathbf{K}_{h} \mathbf{u}_{h}=\left[\mathbf{u}_{h}\right]_{\bar{h}}^{T} \mathbf{K}_{\bar{h}}\left[\mathbf{u}_{h}\right]_{\bar{h}}=\left\|\left[\mathbf{u}_{h}\right]_{\tilde{h}}\right\|^{2} .
$$

\section{Numerical examples}

Three examples are shown to demonstrate the ability of the presented error estimation strategy to drive adaptive computations.

\subsection{Example 1: Spherical dome with vertical distributed load}

The behaviour of the previously introduced error estimator is tested in a problem due to Cirak and Ramm, see [CIR 98]. A detailed description of the geometry can be 


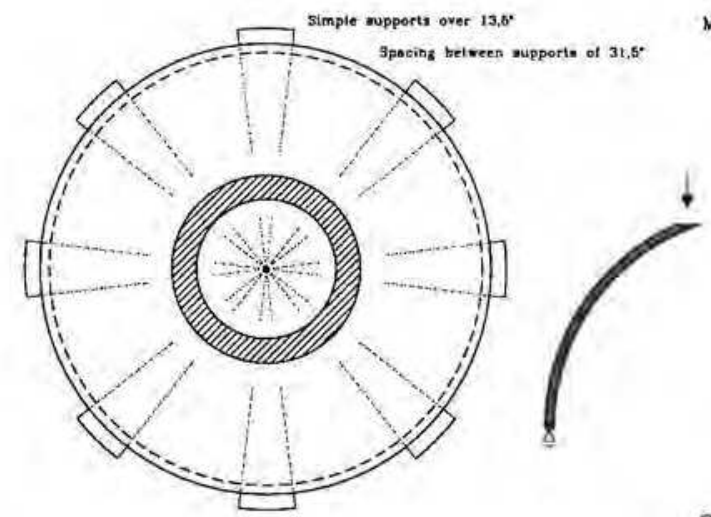

TOP VIEW
Material and foad data

$E=1,092 \mathrm{MPa}$

$t=1,0 \mathrm{~m}$

$p=1.0 \mathrm{~N} / \mathrm{m}$

$v=0.3$

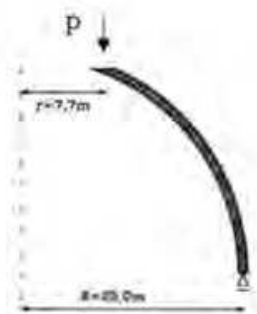

SIDE VIEW

Figure 4. Description of example I

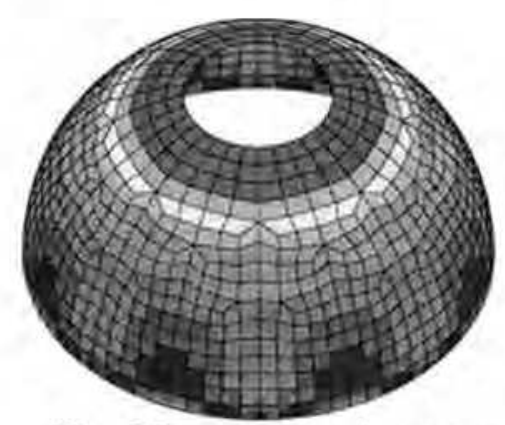

Von Mises comparison stress

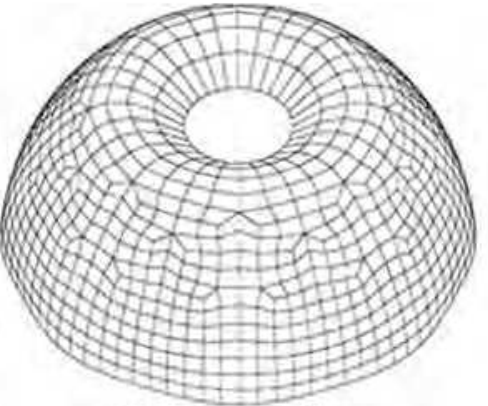

Deformed shape

Figure 5. Solution of the vertically loaded dome

seen in figure 4.

The structure is a semispherical dome with a circular opening in the top vertically loaded on the upper ring. Due to the spaced supports the structure is not completely axisymmetrical and, consequently, a concentration of the stresses is expected on these supports. In figure 5 the distribution of the Von Mises stress computed with a coarse roughly uniform mesh is shown.

The structure has four axes of symmetry, it is enough to consider a computational domain of an eighth of the whole structure. Then, appropriate boundary conditions must be applied on artificial contours that appear in the computational domain.

In order to confirm the efficiency of the error estimator, the distribution of the reference error is compared with the distribution of the estimated error. Recall that the error estimator approximates the reference error. It can be seen in figure 6 that the 


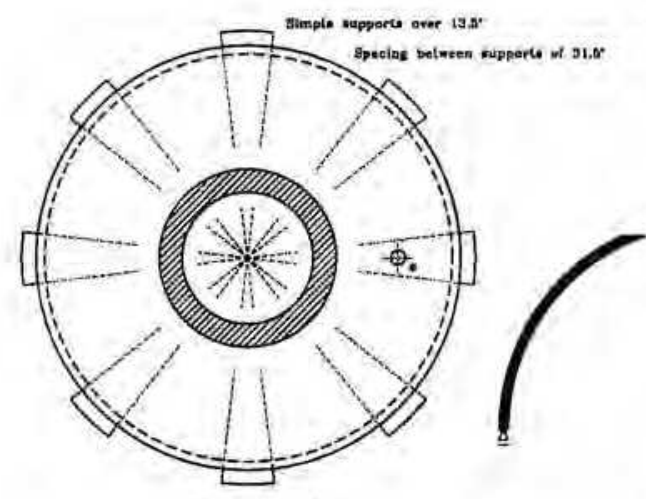

TOP VIEW
Materiel and load dato

$B=1.092 \mathrm{YPC}$

$t=1.0 \mathrm{~m}$

$a=1.0 \mathrm{~m}$

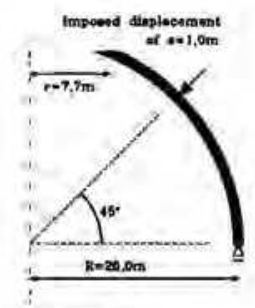

SIDE VIEW

Figure 7. Description of example 2

\subsection{Example 2: Spherical dome with concentrated load}

Example 2 takes the same structure of example 1 but with a singular point load, see figure 7. In this case, due to the location of the load, one half of the structure must be simulated. The adaptive procedure generates a succession of meshes with a concentration of elements in the zone where the load is applied, see figure 8.

\subsection{Example 3: Cooling tower shaped shell}

Example 3 is a shell adopting the shape of an hyperboloid (cooling tower like geometry), the description of the supports and loads is shown in figure 10. In this case, due to the location of the load and supports, one fourth of the structure must be simulated. The adaptive procedure generates a succession of meshes with a concentration of elements in the zone where the load is applied (the top) and the supports, see figure 9.

\section{Conclusions}

A residual type error estimator is generalized to deal with shell elements. This generalization allows easily to account in the error estimation process for the error in the discretization of the geometry. The residual must be carefully computed due to the particular Midlin-type elements used to overcome the transverse shear locking problem. A general procedure is introduced that allows to transfer a solution from one mesh to another in this context. 


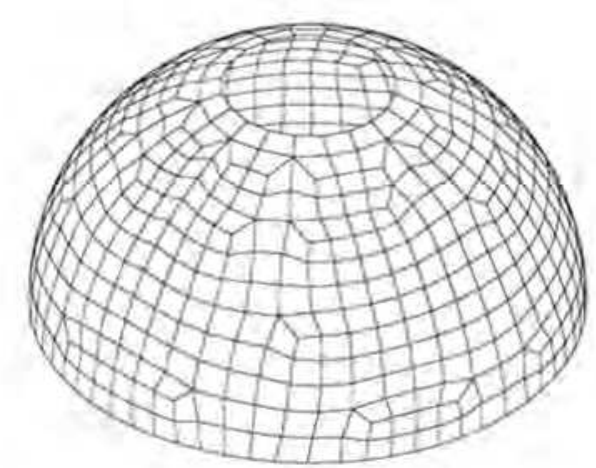

mesh 0: 320 el $59.2 \%$

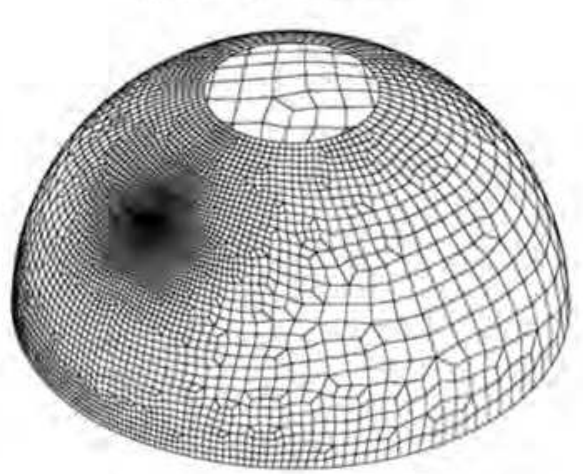

mesh 1: 2242 el $15.1 \%$

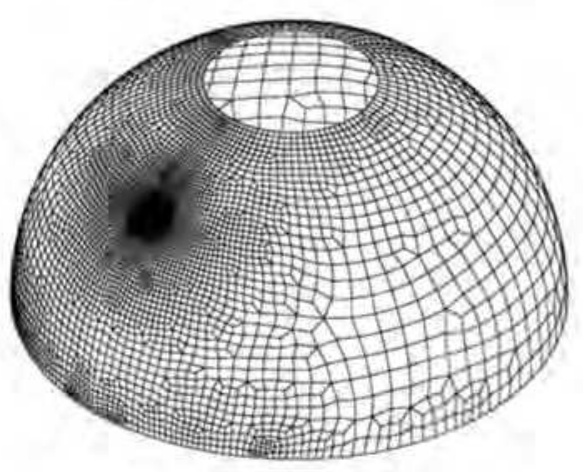

mesh 2: 2698 el $14.0 \%$

Figure 8. Results of the adaptive process in example 2: for each mesh the number of elements and the percentage of error are indicated 


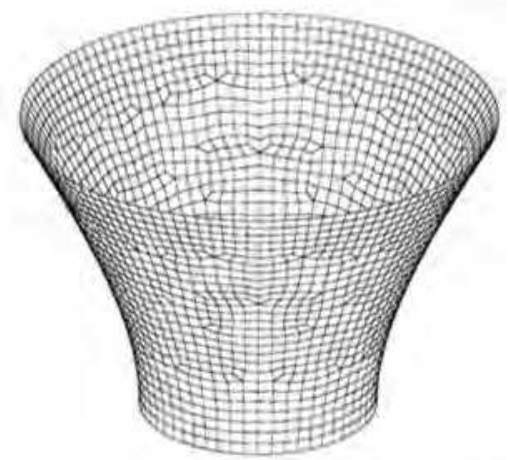

mesh 0: 283 el $35.3 \%$

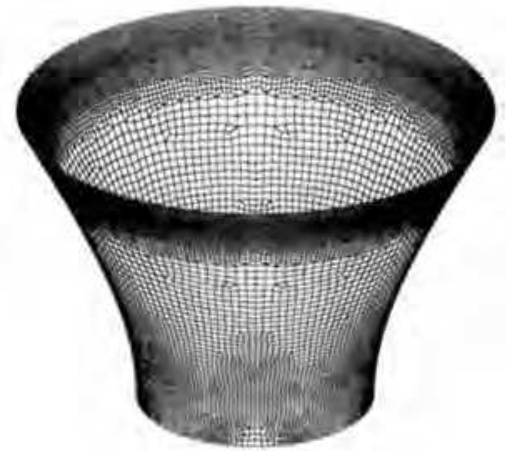

mesh 1: $2388 \mathrm{el} 12.0 \%$

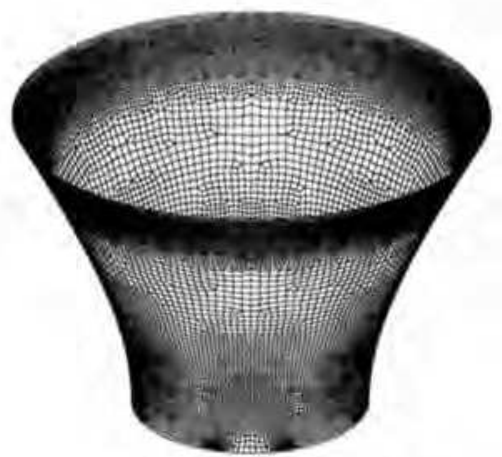

mesh 2: 3232 el $9.6 \%$

Figure 9. Results of the adaptive process in example 3: for each mesh the number of elements and the percentage of error are indicated 


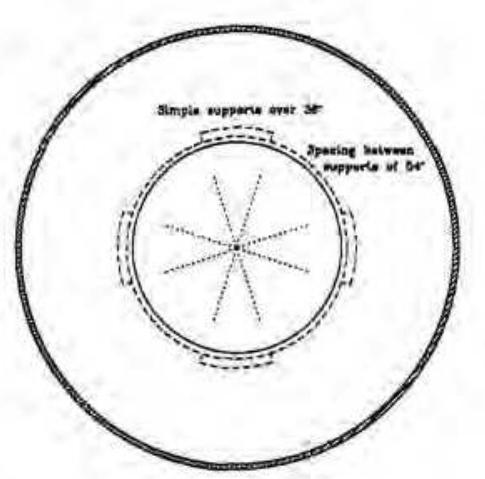

TOP VIEW

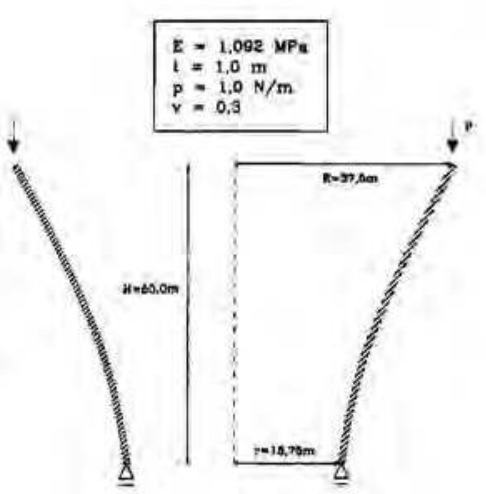

SIDE VIEW

Figure 10. Description of example 3

\section{References}

[AYA 98] AYAD R., DHATT G., BATOZ J.L., "A new hybrid-mixed variational approach for Reissner-Midlin plates. The MiSP model", International Journal for Numerical Methods in Engineering, vol. 42, 1999, p. 1149-1179.

[BAU 97] BAUMANN M., SChWEIZERHOF K., "Adaptive mesh generation of arbitrarily curved shell structures", Computers \& Structures, vol. 64, 1997, p. 209-220.

[CIR 98] CIRAK F., RAMM E., "A-posteriori error estimation and adaptivity for linear elasticity using the reciprocal theorem", Computer Methods in Applied Mechanics and Engineering, vol. 156,1998 , p. $351-362$.

[DIE 97] DFEZ P., EGOZCUE J.J., HUERTA A., "Analysis of the average efficiency of an error estimator", Element Methods: Superconvergence, Post-processing and a Posteriori Error Estimates", M. Křižek et al. eds, Marcel Dekker, New York, 1997, p. 113-126.

[DIE 98] DÍEz P., Egozcue J.J., HuerTA A., "A posteriori error estimation for standard finite element analysis", Computer Methods in Applied Mechanics and Engineering. vol. 163, 1998, p. 141-157.

[DIE 00] DIEz P., ARROYo M., HuerTA A., "Adaptivity based on error estimation for viscoplastic softening materials", Mechanics of Cohesive-Frictional Materials, vol. 5, 2000, p. $87-112$.

[DON 87] DONEA J., LAMAIN L.G., "A modified representation of transverse shear in $\mathcal{C}^{0}$ quadrilateral plate elements", Computer Methods in Applied Mechanics and Engineering, vol. 63,1987 , p. 183-207.

[HUE 97] HUERTA A., DiEz P., Egozcue J.J., "Error estimation for linear and nonlineat problems", Finite Element Methods: Superconvergence, Post-processing and a Posteriori Error Estimates, M. Kŕžžek et al. eds, Marcel Dekker, New York, 1997, p. 183-194.

[HUE 00] HUERTA A., DFEz P., "Error estimation including pollution assessment for nonlinear finite element analysis", Computer Methods in Applied Mechanics and Engineering, vol. 181,2000 , p. $21-41$. 
[HUG 98] HUGHES T.J.R., FEIJÓO G.R., MAZZE1 L., QUINCY J.B., "The variational multiscale method-a paradigm for computational mechanics", Computer Methods in Applied Mechanics and Engineering, vol. 166, 1998, p. 3-24.

[LEE 99] LEE C.K., SZE K.Y., LO S.H., "On using degenerated solid shell elements in adaptive refinement analysis", Intemational Journal for Numerical Methods in Engineering, vol. 45,1999 , p. $627-659$.

[LI 95] Li L.Y., BetTESS P., "Notes on mesh optimal criteria in adaptive finite element computations", Communications in Numerical Methods in Engineering, vol, 11, 1995, p. 911-915.

[PER 96] PERIĆ D., HOCHARD CH., DUTKO M., OWEN D.R.J., "Transfer operators for evolving meshes in small strain elasto-plasticity", Computer Methods in Applied Mechanics and Engineering, vol. 137, 1996, p. 331-344.

[RAV 83] RAVIART P.A., THOMAS J.M., Introduction à l'analyse numérique des équations aux dérivées partielles, Masson, Paris, 1983.

[RIC 97] RICCIUS J., SCHWEIZERHOF K., BAUMANN M., "Combination of adaptivity and mesh smoothing for the finite element analysis of shell intersections", International Joumal for Numerical Methods in Engineering, vol. 40, 1997, p. 2459-2474. 